\title{
The role of delta radiomics in gastric cancer
}

\author{
Maria Antonietta Mazzei ${ }^{1}$, Valerio Nardone ${ }^{2}$, Letizia Di Giacomo ${ }^{1}$, Giulio Bagnacci ${ }^{1}$, Francesco Gentili ${ }^{1}$, \\ Paolo Tini $^{2}$, Daniele Marrelli ${ }^{3}$, Luca Volterrani ${ }^{1}$
}

${ }^{1}$ Department of Medicine, Surgery and Neuroscience, Unit of Diagnostic Imaging, Azienda Ospedaliera Universitaria Senese, University of Siena, Siena, Italy; ${ }^{2}$ Unit of Radiation Oncology, Azienda Ospedaliera Universitaria Senese, Siena, Italy; ${ }^{3}$ Department of Medicine, Surgery and Neuroscience, Unit of General Surgery and Surgical Oncology, University of Siena, Siena, Italy

Correspondence to: Francesco Gentili, MD. Department of Medicine, Surgery and Neuroscience, Unit of Diagnostic Imaging, Azienda Ospedaliera Universitaria Senese, University of Siena, Siena, Italy. Email: francescogentili@gmail.com.

Submitted Jul 20, 2018. Accepted for publication Jul 27, 2018.

doi: 10.21037/qims.2018.07.08

View this article at: http://dx.doi.org/10.21037/qims.2018.07.08

We have read with great interest and appreciated the paper by Hou et al. entitled "Radiomic analysis using contrastenhanced computed tomography (CT): predict treatment response to pulsed low dose rate radiotherapy in gastric carcinoma with abdominal cavity metastasis" (1).

The authors successfully tried to correlate radiomic features, calculated on three-dimensional 3D VOI'S drawn slightly within the border of the tumoral lesions, with therapeutic response assessed according to the response evaluation criteria in solid tumors (RECIST v1.1). In particular, they found that 6 features (1 first order-based, 1 texture-based, 1 log-based, and 3 wavelet-based) were significantly different between responders (patients with complete or partial response) and non-responders (patients with stable or progressive disease). These findings suggest that radiomic analysis of contrast-enhanced CT images may provide additional information regarding therapeutic response, which could be important for the clinical decision-making process.

In fact, texture analysis (TA) of CT images seems to be able to detect subtle differences in CT values which cannot be recognized by human eyes, providing quantitative data on tumour phenotype and microenvironment by analyzing the relation, distribution and relationship of pixel intensities (2).

Another therapeutic option for metastatic gastric cancer (GC), which has spread during the last years, is conversion surgery, defined as a surgical treatment aiming at R0 resection after chemotherapy in patients with originally unresectable or only marginally resectable advanced gastric cancer (AGC) (3). For this reason, neoadjuvant chemotherapy (NAC) still remains a standard of care both for metastatic and locally AGC and an accurate evaluation of overall tumour burden and discrimination between responders and non-responders, prior to surgery, has than become increasingly important to customize patient treatment and reduce healthcare costs, delivering aggressive multimodal treatments only to patients who potentially might benefit from these $(4,5)$.

In this sense it is rising interest to identify surrogate imaging markers for predicting tumour histopathological regression prior to surgery and CT studies have shown promising results for improving patient stratification, by using different approach, such as linear or volumetric measurement systems or radiomic analysis (6-8).

In our experience, delta radiomics, defined as the calculation of the modification of texture parameters before and after NAC, has shown the potentiality to predict histopathological response and patient outcome, overcoming the limit of a model based only on pre or posttreatment radiomic features (9). We have preliminary analyzed a homogeneous cohort of 23 patients with biopsy proven resectable AGC ( $\geq \mathrm{T} 3$ or $\mathrm{N}+)$, treated with NAC and radical surgery. Gastric lesion, defined as gross tumour volume (GTV) was contoured on every slice of pre and post NAC contrast enhanced CT scan, obtaining a $3 \mathrm{D}$ region of interest (3D-ROI) with the contouring software RayStation, using a method already validated in different settings (10). Finally, a correlation between the texture parameters [histogram, shape and grey-level co-occurrence matrix (GLCM)] extracted from the 3D-ROI and the complete 

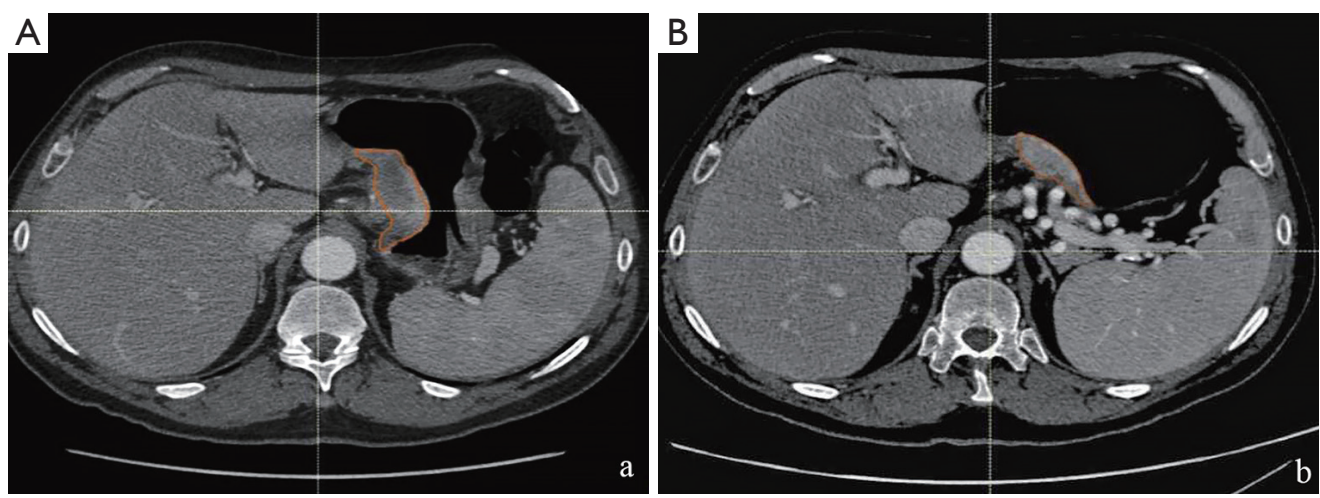

Figure 1 A 67-year-old man with advanced gastric cancer of the lesser curvature who underwent contrast enhanced CT pre (A) and post (B) NAC. Although the post-NAC CT clearly shows a reduction in the size of tumoral mass, delta parameters (entropy, GLCM-contrast, GLCM-entropy, GLCM-dissimilarity) classify this patient as non-responder. The finding is confirmed on final histopathology after surgery (adenocarcinoma ypT3, tumor regression grade 3 according to Becker et al.). NAC, neoadjuvant chemotherapy; CT, computed tomography; GLCM, grey-level co-occurrence matrix.

pathological response (ypCR: tumour regression grade 1 according to Becker et al.) was searched. In our preliminary case population only three patients showed a ypCR, and only delta parameters entropy $(\mathrm{P}=0.003)$, GLCM-contrast ( $\mathrm{P}<0.001)$, GLCM-entropy $(\mathrm{P}=0.004)$, GLCM-dissimilarity $(\mathrm{P}=0.001)$ were correlated with this outcome at univariate analysis whereas at multivariate analysis, the only parameter that showed a significant correlation was the delta GLCMcontrast $\left(R^{2}: 0.539\right.$, sensibility $66 \%$, specificity $100 \%$, AUC 0.733) (Figure 1).

We are going to test these results with external validation, from a wider cohort of about 100 patients coming from different centres of the Italian Research Group for Gastric Cancer (GIRCG).

\section{Acknowledgements}

None.

\section{Footnote}

Conflicts of Interest: The authors have no conflicts of interest to declare.

\section{References}

1. Hou Z, Yang Y, Li S, Yan J, Ren W, Liu J, Wang K, Liu B, Wan S. Radiomic analysis using contrast-enhanced CT: predict treatment response to pulsed low dose rate radiotherapy in gastric carcinoma with abdominal cavity metastasis. Quant Imaging Med Surg 2018;8:410-20.

2. Lubner MG, Smith AD, Sandrasegaran K, Sahani DV, Pickhardt PJ. CT Texture Analysis: Definitions, Applications, Biologic Correlates, and Challenges. Radiographics 2017;37:1483-503.

3. Yoshida K, Yamaguchi K, Okumura N, Tanahashi T, Kodera Y. Is conversion therapy possible in stage IV gastric cancer: the proposal of new biological categories of classification. Gastric Cancer 2016;19:329-38.

4. De Manzoni G, Marrelli D, Baiocchi GL, Morgagni P, Saragoni L, Degiuli M, Donini A, Fumagalli U, Mazzei MA, Pacelli F, Tomezzoli A, Berselli M, Catalano F, Di Leo A, Framarini M, Giacopuzzi S, Graziosi L, Marchet A, Marini M, Milandri C, Mura G, Orsenigo E, Quagliuolo V, Rausei S, Ricci R, Rosa F, Roviello G, Sansonetti A, Sgroi G, Tiberio GA, Verlato G, Vindigni C, Rosati R, Roviello F. The Italian Research Group for Gastric Cancer (GIRCG) guidelines for gastric cancer staging and treatment: 2015. Gastric Cancer 2017;20:20-30.

5. Mazzei MA, Khader L, Cirigliano A, Cioffi Squitieri N, Guerrini S, Forzoni B, Marrelli D, Roviello F, Mazzei FG, Volterrani L. Accuracy of MDCT in the preoperative definition of peritoneal cancer index (PCI) in patients with advanced ovarian cancer who underwent peritonectomy and hyperthermic intraperitoneal chemotherapy (HIPEC). Abdominal Imaging 2013;38:1422-30.

6. Mazzei MA, Bagnacci G, Gentili F, Nigri A, Pelini V, Vindigni C, Mazzei FG, Baiocchi GL, Pittiani F, Morgagni P, Petrella E, Mura G, Verdelli B, Bencivenga M, Giacopuzzi S, Marrelli D, Roviello F, Volterrani L. Gastric 
Cancer Maximum Tumour Diameter Reduction Rate at CT Examination as a Radiological Index for Predicting Histopathological Regression after Neoadjuvant Treatment: A Multicentre GIRCG Study. Gastroenterol Res Pract 2018;2018:1794524.

7. Giganti F, Marra P, Ambrosi A, Salerno A, Antunes S, Chiari D, Orsenigo E, Esposito A, Mazza E, Albarello L, Nicoletti R, Staudacher C, Del Maschio A, De Cobelli F. Pre-treatment MDCT-based texture analysis for therapy response prediction in gastric cancer: Comparison with tumour regression grade at final histology. Eur J Radiol 2017;90:129-37.

8. Lee SM, Kim SH, Lee JM, Im SA, Bang YJ, Kim WH, Kim MA, Yang HK, Lee HJ, Kang WJ, Han JK, Choi

Cite this article as: Mazzei MA, Nardone V, Di Giacomo L, Bagnacci G, Gentili F, Tini P, Marrelli D, Volterrani L. The role of delta radiomics in gastric cancer. Quant Imaging Med Surg 2018;8(7):719-721. doi: 10.21037/qims.2018.07.08
BI. Usefulness of CT volumetry for primary gastric lesions in predicting pathologic response to neoadjuvant chemotherapy in advanced gastric cancer. Abdom Imaging 2009;34:430-40.

9. Fave X, Zhang L, Yang J, Mackin D, Balter P, Gomez D, Followill D, Jones AK, Stingo F, Liao Z, Mohan R, Court L. Delta-radiomics features for the prediction of patient outcomes in non-small cell lung cancer. Sci Rep 2017;7:588.

10. Nardone V, Tini P, Nioche C, Mazzei MA, Carfagno T, Battaglia G, Pastina P, Grassi R, Sebaste L, Pirtoli L. Texture analysis as a predictor of radiation-induced xerostomia in head and neck patients undergoing IMRT. Radiol Med 2018;123:415-23. 